Mathematical Research Letters 3, 103-107 (1996)

\title{
GALOIS REPRESENTATIONS IN THE TATE-SHAFAREVICH GROUP OF AN ELLIPTIC CURVE
}

\author{
DAVID E. ROHRLICH
}

This letter is intended as a footnote to a paper of Kramer [2]. The main result of $[2]$ is that there exist semistable elliptic curves $E$ over $\mathbb{Q}$ with arbitrarily large $\amalg(E / \mathbb{Q})_{2}$, where $\amalg(E / \mathbb{Q})$ is the Tate-Shafarevich group of $E$ over $\mathbb{Q}$ and $\amalg(E / \mathbb{Q})_{2}$ the subgroup of elements of order dividing 2 . The footnote to be added here is that Kramer's construction also gives Tate-Shafarevich groups which are arbitrarily large as Galois-modules:

Theorem. Let $K$ be a finite Galois extension of $\mathbb{Q}$ and $n$ a positive integer. There exists a semistable elliptic curve $E$ over $\mathbb{Q}$ such that the natural representation of $\operatorname{Gal}(K / \mathbb{Q})$ on $\amalg(E / K)_{2}$ contains a subrepresentation isomorphic to the direct sum of $n$ copies of the regular representation of $\operatorname{Gal}(K / \mathbb{Q})$ over $\mathbb{F}_{2}$.

Let $m$ be an integer. As in [2], we consider the elliptic curves

$$
A: y^{2}+x y=x^{3}+8 m x^{2}+m(16 m+1) x
$$

and

$$
B: y^{2}+x y=x^{3}-16 m x^{2}-8 m x-m
$$

over $\mathbb{Q}$. They are related by an isogeny $f: A \rightarrow B$ with kernel the group of order 2 generated by the point $(0,0)$ on $A$. Let $\operatorname{Sel}_{2}(A / K)$ and $\operatorname{Sel}_{g}(B / K)$ denote respectively the 2-Selmer group of $A$ over $K$ and the $g$-Selmer group of $B$ over $K$, where $g: B \rightarrow A$ is the isogeny dual to $f$. Since $f \circ g$ is multiplication by 2 , the kernel of the $\operatorname{map} \amalg(B / K) \rightarrow \amalg(A / K)$ induced by $g$ is a subgroup $\amalg(B / K)_{g}$ of $\amalg(B / K)_{2}$. A key role in Kramer's argument is played by the commutative diagram

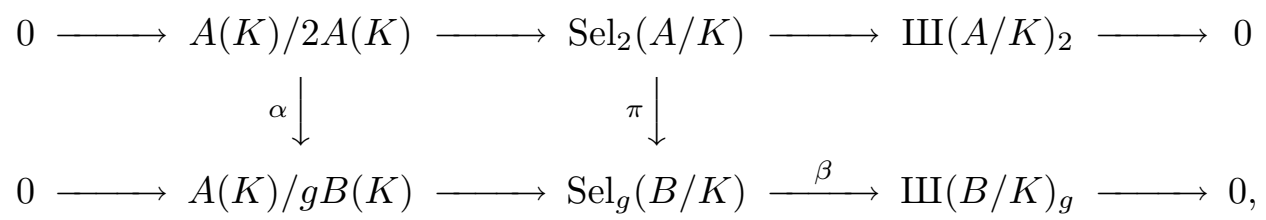

Received November 1, 1995.

Research partially supported by NSF grant DMS-9396090 and the Mathematical Sciences Research Institute. 
where $\alpha$ is surjective and the rows are exact. If $V$ is a subspace of $\operatorname{Sel}_{g}$ $(B / K)$ which intersects the image of $\pi$ trivially then $\beta$ maps $V$ injectively into $\amalg(B / K)_{g}$, because $\alpha$ is surjective. We shall see that for an appropriate choice of $m$ there exists such a subspace $V$ which is stable under $\operatorname{Gal}(K / \mathbb{Q})$ and isomorphic as a representation to the direct sum of $n$ copies of the regular representation.

Our choice of $m$ differs slightly from Kramer's in that we take $m$ even rather than odd. The reason for this change is that we want $A$ to have split multiplicative reduction at 2 rather than ordinary reduction so that we will be able to apply the local results of Brumer-Kramer [1] even when $K$ is ramified at 2 . The 2-Selmer group of an elliptic curve with ordinary reduction over a ramified extension of $\mathbb{Q}_{2}$ does not seem to have been determined as yet.

We denote the completion of $K$ at a place $v$ by $K_{v}$. If $v$ is the finite place corresponding to a prime ideal $\mathfrak{m}$ then we write $v=v(\mathfrak{m})$. If $v=v(\mathfrak{m})$ and $\mathfrak{m}$ divides $m$ then we also write $v \mid m$.

Lemma. Given a positive integer $n$, there exists a positive even integer $m$ with the following properties:

(i) There are at least $n$ distinct primes $m_{1}, m_{2}, \ldots, m_{n}$ which divide $m$ and split completely in $K$.

(ii) The integer $\ell=16 m+1$ has the form

$$
\ell=\ell_{1} \ell_{2} \ldots \ell_{n} r
$$

where $r$ is a prime congruent to $1(\bmod 4)$ and $\ell_{1}, \ell_{2}, \ldots, \ell_{n}$ are distinct primes congruent to $1(\bmod 4)$ which split completely into principal prime ideals of $K$.

(iii) Suppose that the principal fractional ideal generated by an element $b \in K^{\times}$is the square of an ideal of $K$. Then $b \in K_{v}^{\times 2}$ for $v \mid m_{i}(1 \leqslant i \leqslant n)$. Furthermore, there exist

- prime ideals $\mathfrak{m}_{1}, \mathfrak{m}_{2}, \ldots, \mathfrak{m}_{n}$ of $K$ lying over $m_{1}, m_{2}, \ldots, m_{n}$ respectively,

- prime ideals $\mathfrak{l}_{1}, \mathfrak{l}_{2}, \ldots, \mathfrak{l}_{n}$ of $K$ lying over $\ell_{1}, \ell_{2}, \ldots, \ell_{n}$ respectively, and

- generators $\lambda_{1}, \lambda_{2}, \ldots, \lambda_{n}$ of $\mathfrak{l}_{1}, \mathfrak{l}_{2}, \ldots, \mathfrak{l}_{n}$ respectively

such that for $1 \leqslant i, j \leqslant n$ and $\sigma, \tau \in \operatorname{Gal}(K / \mathbb{Q})$,

$$
\lambda_{i}^{\sigma} \notin K_{v}^{\times 2} \text { with } v=v\left(\mathfrak{m}_{j}^{\tau}\right) \Longleftrightarrow i=j \text { and } \sigma=\tau .
$$

Proof. The proof is much the same as in [2], but with the additional input of the Chebotarev density theorem. 
Let $H$ be the Hilbert class field of $K$ and let $\ell_{1}, \ell_{2}, \ldots, \ell_{n}$ be distinct primes which split completely in $H(\sqrt{-1})$. Then the $\ell_{i}$ split completely in $K$ and are congruent to $1(\bmod 4)$ because they split in $\mathbb{Q}(\sqrt{-1})$. Let $\mathfrak{l}_{i}$ be any prime ideal of $K$ lying over $\ell_{i}$. Since $\mathfrak{l}_{i}$ splits completely in $H$ it is a principal ideal, and we let $\lambda_{i}$ be any generator.

Next choose a set of fractional ideals of $K$ which represent the distinct ideal classes of order 2 , and for each representative $\mathfrak{b}$ choose a generator $b(\mathfrak{b})$ of the principal ideal $\mathfrak{b}^{2}$. Let $B$ be the set of elements $b(\mathfrak{b})$ so obtained, and let $U$ be a set of generators of the group of integral units of $K$. Then the extension $F$ of $K$ defined by

$$
F=K(\{\sqrt{z}: z \in B \cup U\})
$$

is independent of any choices and hence Galois over $\mathbb{Q}$. Put

$$
\Lambda=\left\{\lambda_{i}^{\sigma}: 1 \leqslant i \leqslant n, \sigma \in \operatorname{Gal}(K / \mathbb{Q})\right\} .
$$

Since the $\ell_{i}$ are odd primes while the extension $F / K$ is unramified outside 2 and infinity, the extension

$$
L=F(\{\sqrt{\lambda}: \lambda \in \Lambda\})
$$

has the same degree over $F$ as $K(\{\sqrt{\lambda}: \lambda \in \Lambda\})$ has over $K$, namely $2^{n[K: \mathbb{Q}]}$. In particular, putting

$$
\Lambda_{j}=\Lambda-\left\{\lambda_{j}\right\}
$$

and

$$
L_{j}=F\left(\left\{\sqrt{\lambda}: \lambda \in \Lambda_{j}\right\}\right),
$$

we have $\left[L: L_{j}\right]=2$ for $1 \leqslant j \leqslant n$. Let $\mathfrak{M}_{1}, \mathfrak{M}_{2}, \ldots, \mathfrak{M}_{n}$ be primes ideals of $L$, unramified over $\mathbb{Q}$, such that the Artin symbol $\left(\mathfrak{M}_{j}, L / \mathbb{Q}\right)$ coincides with the nontrivial element of $\operatorname{Gal}\left(L / L_{j}\right)$. Put $\mathfrak{m}_{j}=K \cap \mathfrak{M}_{j}$ and let $m_{j}$ be the rational prime lying below $\mathfrak{m}_{j}$. If $\mathfrak{m}$ is a prime ideal of $K$ lying over some $m_{j}$, then $\mathfrak{m}$ splits completely in $F$ and consequently $b \in K_{v}^{\times 2}$ when $v=v(\mathfrak{m})$ and $b$ is as in (iii). Furthermore, for $v=v\left(\mathfrak{m}_{j}\right)$ we have $\lambda_{i}^{\sigma} \notin K_{v}^{\times 2}$ if and only if $i=j$ and $\sigma=1$, whence the second assertion in (iii) follows on replacing $\sigma$ by $\sigma \tau^{-1}$.

To complete the proof of the lemma, choose a prime $r$ such that

$$
\ell_{1} \ell_{2} \ldots \ell_{n} r \equiv 1 \quad\left(\bmod 32 m_{1} m_{2} \ldots m_{n}\right) \text {, }
$$

and put $m=\left(\ell_{1} \ell_{2} \ldots \ell_{n} r-1\right) / 16$. Then $m$ is a positive even integer with the required properties, and $\ell=1+16 \mathrm{~m}$ has the required form. 
Let $\mathcal{L}$ and $\mathcal{M}$ be the sets of prime divisors of $\ell$ and $m$ respectively, where $\ell$ and $m$ are as in the lemma. Then $A$ has good reduction outside $\mathcal{L} \cup \mathcal{M}$ and split multiplicative reduction at each prime in $\mathcal{L} \cup \mathcal{M}$ (cf. Lemma 1 of [2] - the key point is that the number $b_{2}=1+32 m=2 \ell-1$ belongs to $\mathbb{Q}_{p}^{\times 2}$ for every $p \in \mathcal{L} \cup \mathcal{M}$, because if $p \in \mathcal{L}$ then $\left.p \equiv 1(\bmod 4)\right)$. We may therefore use the results of Brumer-Kramer [1] to calculate the relevant Selmer groups. As in [2], we view $\operatorname{Sel}_{2}(A / K)$ and $\operatorname{Sel}_{g}(B / K)$ as subgroups

$$
\operatorname{Sel}_{2}(A / K) \subset \prod_{\nu=1}^{3}\left(K^{\times} / K^{\times 2}\right)
$$

and

$$
\operatorname{Sel}_{g}(B / K) \subset K^{\times} / K^{\times 2} .
$$

The map $\pi: \operatorname{Sel}_{2}(A / K) \rightarrow \operatorname{Sel}_{g}(B / K)$ is then identified with the restriction to $\operatorname{Sel}_{2}(A / K)$ of the projection map

$$
\begin{aligned}
\prod_{\nu=1}^{3}\left(K^{\times} / K^{\times 2}\right) & \rightarrow K^{\times} / K^{\times 2} \\
{[a, b, c] } & \mapsto[a],
\end{aligned}
$$

where the square brackets denote the class modulo squares of the triple $(a, b, c)$ or the element $a$. Given a finite place $v$ of $K$, let $\mathcal{O}_{v}$ denote the ring of integers of $K_{v}$. Arguing as in Lemma 2 of [2], but omitting part (ii) of the lemma and observing that the restriction " $p$ odd" in part (iii) is unnecessary, we find that $\operatorname{Sel}_{2}(A / K)$ consists of all classes $[a, b, a b]$ satisfying the following conditions:

(1) If $v$ is finite but $v \nmid \ell m$ then $a, b \in K_{v}^{\times 2} \mathcal{O}_{v}^{\times}$

(2) If $v \mid m$ then $b \in a K_{v}^{\times 2}$.

(3) If $v \mid \ell$ then $b \in K_{v}^{\times 2}$.

(4) If $K_{v}=\mathbb{R}$ then $b \in a K_{v}^{\times 2}$.

In addition, the remark following the proof of Lemma 2 on p. 382 of [2] shows that $\operatorname{Sel}_{g}(B / K)$ consists of all classes $[a]$ such that $a \in K_{v}^{\times 2} \mathcal{O}_{v}^{\times}$for finite $v \nmid \ell m$.

Now let $V$ be the subgroup of $K^{\times} / K^{\times 2}$ generated by the classes $\left[\lambda_{i}^{\sigma}\right]$ for $1 \leqslant i \leqslant n$ and $\sigma \in \operatorname{Gal}(K / \mathbb{Q})$, where the $\lambda_{i}$ are as in the lemma. Then $V$ is contained in $\operatorname{Sel}_{g}(B / K)$ and the representation of $\operatorname{Gal}(K / \mathbb{Q})$ on $V$ is isomorphic to the direct sum of $n$ copies of the regular representation. We claim that $V$ intersects the image of $\pi$ trivially. Indeed, suppose that 
$[a, b, a b] \in \operatorname{Sel}_{2}(A / K)$ and $[a] \in V$. After replacing $a$ by another element of $a K^{\times 2}$, we may assume that $a$ has the form

$$
a=\prod_{\substack{1 \leqslant i \leqslant n \\ \sigma \in \operatorname{Gal}(K / \mathbb{Q})}}\left(\lambda_{i}^{\sigma}\right)^{\epsilon(i, \sigma)}
$$

with $\epsilon(i, \sigma)=0$ or 1 for all $i$ and $\sigma$. In particular, $a \in \mathcal{O}_{v}^{\times}$for $v \mid m$. Condition (2) above then implies that $b \in K_{v}^{\times 2} \mathcal{O}_{v}^{\times}$for $v \mid m$. But by conditions (1) and (3) we also have $b \in K_{v}^{\times 2} \mathcal{O}_{v}^{\times}$for finite $v \nmid m$. Hence the principal ideal generated by $b$ is the square of an ideal of $K$. Therefore $b \in K_{v}^{\times 2}$ whenever $v$ has the form $v=v\left(\mathfrak{m}_{j}^{\tau}\right)$ for some $j$ and $\tau$. For all such $v$ condition (2) then gives $a \in K_{v}^{\times 2}$. It follows that $\epsilon(i, \sigma)=0$ for all $i$ and $\sigma$, whence $[a]$ is trivial.

\section{Acknowledgement}

The original version of this note (submitted before I was aware of Kramer's beautiful paper) pertained only to $\operatorname{Sel}_{2}(E / K)$, not to $\amalg(E / K)_{2}$. My attention was drawn to [2] by the referee, for whose knowledgeable comments I am deeply grateful.

\section{References}

1. A. Brumer and K. Kramer, The rank of elliptic curves, Duke Math. J. 44 (1977), 715-743.

2. K. Kramer, A family of semistable elliptic curves with large Tate-Shafarevitch groups, Proc. AMS 89 (1983), 379-386.

Department of Mathematics, Boston University, Boston, M A 02215

E-mail address: rohrlich@math.bu.edu 